Supporting Information

\title{
Molecularly Imprinted Phase-Change Microcapsule System for Bifunctional Applications in Waste Heat Recovery and Targeted Pollutant Removal
}

Jinfei Niu ${ }^{\dagger}$, Huan $\mathrm{Liu}^{\dagger}$, Xiaodong Wang*, Dezhen Wu

State Key Laboratory of Organic-Inorganic Composites, Beijing University of Chemical Technology, Beijing 100029, China.

$\dagger^{T}$ The two authors made equal contributions to this work.

* Corresponding authors: Tel \& Fax: +86 106442 1693. E-mail: wangxdfox@aliyun.com (X. Wang). 


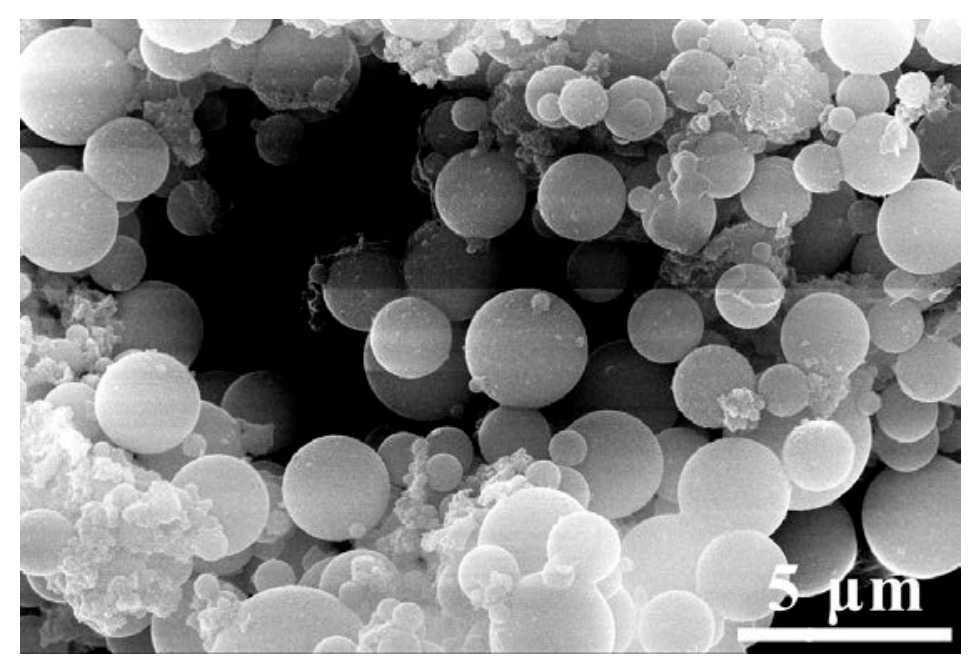

Figure S1. SEM micrographs of the MIMs after 500 cycles of heating-cooling cyclic treatments. 


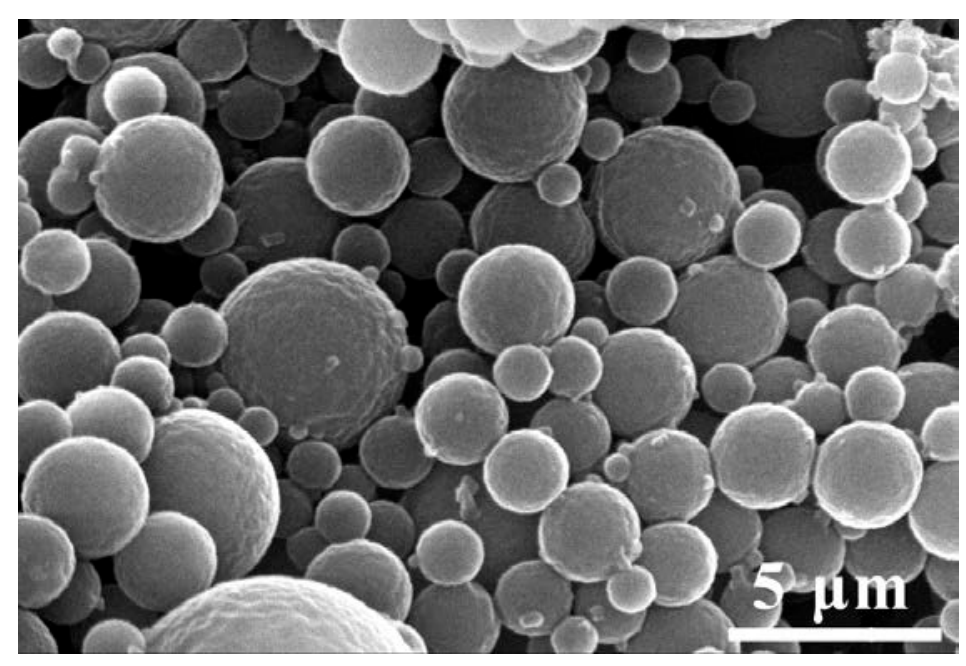

Figure S2. SEM micrograph of the NIMs. 


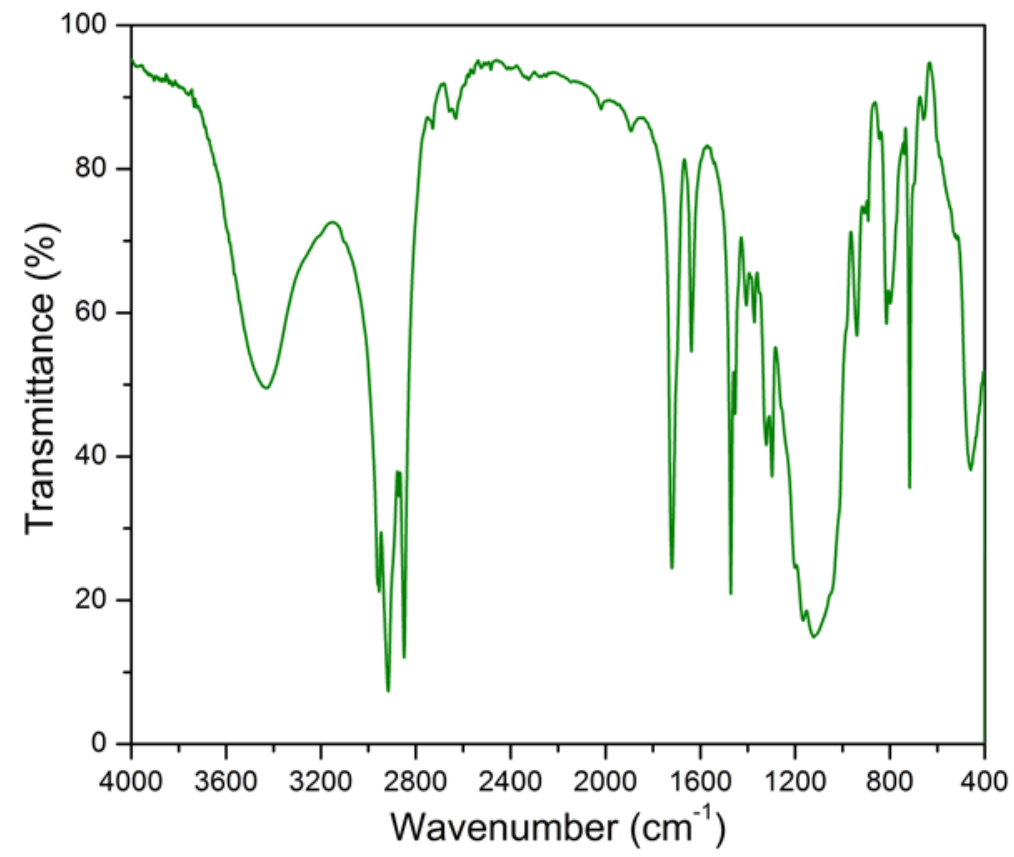

Figure S3. FTIR spectrum of the NIMs. 
Table S1. The thermal energy-storage performance obtained from DSC measurements for the MIMs and NIMs.

\begin{tabular}{lllll}
\hline Sample & $\begin{array}{l}T_{\mathrm{c}} \\
\left({ }^{\circ} \mathrm{C}\right)\end{array}$ & $\begin{array}{l}T_{\mathrm{m}} \\
\left({ }^{\circ} \mathrm{C}\right)\end{array}$ & $\begin{array}{l}\Delta H_{\mathrm{c}} \\
(\mathrm{J} / \mathrm{g})\end{array}$ & $\begin{array}{l}\Delta H_{\mathrm{m}} \\
(\mathrm{J} / \mathrm{g})\end{array}$ \\
\hline \multirow{2}{*}{ MIMs } & 29.4 & 37.6 & 164.1 & 165.3 \\
\multirow{2}{*}{ NIMs } & 29.3 & 37.4 & 164.3 & 165.6 \\
\hline
\end{tabular}


Table S2. The equilibrium adsorption capacity of the MIMs at different mass ratios of EGDMA/MAA.

\begin{tabular}{ll}
\hline $\begin{array}{l}\text { Mass ratio of EGDMA/MAA } \\
\text { (wt. / wt.) }\end{array}$ & $\begin{array}{l}\text { Kinetic adsorption capacity at equilibrium } \\
\text { (mg/g) }\end{array}$ \\
\hline $50 / 50$ & 6.54 \\
$60 / 40$ & 9.69 \\
$70 / 30$ & 11.40 \\
$80 / 20$ & 20.35 \\
$90 / 10$ & 15.35 \\
\hline
\end{tabular}


Table S3. The recovery efficiency and thermal energy-storage performance of the MIMs after different rebinding-elution cycles.

\begin{tabular}{|c|c|c|c|c|c|c|c|c|c|}
\hline \multirow[t]{2}{*}{ Sample } & \multicolumn{3}{|c|}{$\begin{array}{l}\text { Recovery efficiency } \\
\text { (\%) }\end{array}$} & \multicolumn{3}{|l|}{$\begin{array}{l}\Delta H_{\mathrm{c}} \\
(\mathrm{J} / \mathrm{g})\end{array}$} & \multicolumn{3}{|l|}{$\begin{array}{l}\Delta H_{\mathrm{m}} \\
(\mathrm{J} / \mathrm{g})\end{array}$} \\
\hline & 1st cycle & 5th cycle & 10th cycle & 1st cycle & 5th cycle & 10th cycle & 1st cycle & 5th cycle & 10th cycle \\
\hline MIMs & 100.0 & 85.0 & 61.7 & 164.3 & 164.2 & 162.9 & 165.2 & 165.3 & 163.2 \\
\hline
\end{tabular}

